\title{
Dietary supplement use and nosebleeds in hereditary haemorrhagic telangiectasia - an observational study
}

\author{
Basel Chamali ${ }^{1,2}$, Helen Finnamore ${ }^{1,3}$, Richard Manning ${ }^{4}$, Michael A Laffan ${ }^{4,5}$, Mary Hickson ${ }^{6}$, \\ Kevin Whelan ${ }^{3}$, Claire L Shovlin ${ }^{1,7, *}$ \\ ${ }^{1}$ National Heart and Lung Institute Cardiovascular Sciences, Imperial College London, UK, \\ ${ }^{2}$ Imperial College School of Medicine, Imperial College London, UK; \\ ${ }^{3}$ Diabetes and Nutritional Sciences Division, King's College London, UK; \\ ${ }^{4}$ Haematology Department, Hammersmith Hospital, Imperial College Healthcare NHS Trust, London, UK; \\ ${ }^{5}$ Centre for Haematology, Imperial College London, UK \\ ${ }^{6}$ Nutrition and Dietetics, Imperial College Healthcare NHS Trust, London, UK (Current address: School of Health Professions, \\ Plymouth University, Plymouth, UK); \\ ${ }^{7}$ HHTIC London, Hammersmith Hospital, Imperial College Healthcare NHS Trust, London, UK.
}

\begin{abstract}
Summary Understanding potential provocations of haemorrhage is important in a range of clinical settings, and particularly for people with abnormal vasculature. Patients with hereditary haemorrhagic telangiectasia (HHT) can report haemorrhage from nasal telangiectasia in real time, and suggested dietary factors may precipitate nosebleeds. To examine further, nosebleed severity, dietary supplement use, and blood indices were evaluated in an unselected group of 50 HHT patients recruited from a specialist UK service. Using the validated Epistaxis Severity Score, nosebleed severity ranged from 0 to 9.1 out of 10 (median 3.9). Using a Food Frequency Questionnaire, 24/50 (48\%) participants reported use of dietary supplements in the previous year. A third $(18 / 50 ; 36 \%)$ had used self prescribed, non-iron containing dietary supplements, ingesting between 1 and 3 different supplements each day. Eight (16\%) used fish oils. Despite having more severe epistaxis $(p=\mathbf{0 . 0 1 2})$, the 12 iron supplement users had higher serum iron concentrations, and were able to maintain their red blood cell indices. In contrast, there was no evident benefit for the participants using non iron supplements. Furthermore, platelet counts and serum fibrinogen tended to be lower in fish oil/supplement users, and one fish oil user demonstrated reduced in vitro platelet aggregation. In conclusion, in this small study, a third of HHT patients used non-iron dietary supplements, and one in six ingested fish oils, unaware of their known anti-platelet activity. The scale of use, and potential of these "natural health supplements" to exacerbate nosebleeds has not been appreciated previously in HHT.
\end{abstract}

Keywords: Epistaxis, Diet, Iron, epistaxis severity score (ESS), fish oils

\section{Introduction}

Patients with hereditary haemorrhagic telangiectasia (HHT) provide an intriguing real-life model to

Released online in J-STAGE as advance publication April 18, 2016.

*Address correspondence to:

Dr. Claire L. Shovlin PhD FRCP, Reader in Clinical and Molecular Medicine, NHLI Cardiovascular Sciences, Imperial Centre for Translational and Experimental Medicine, Imperial College London, Hammersmith Campus, Du Cane Road, London W12 0NN, UK.

E-mail: c.shovlin@imperial.ac.uk evaluate potential precipitants of haemorrhage. Due to a causative mutation most commonly in an ENG, ACVRL1 or SMAD4 gene, HHT leads to the development of vascular abnormalities, particularly visceral arteriovenous malformations, and smaller nasal and gastrointestinal telangiectasia $(1,2)$. Patients with HHT can report nosebleeds (epistaxis) in real time, and the magnitude of their nasal haemorrhagic losses are such that iron deficiency anaemia commonly results, permitting objective evaluations (3).

Understanding nosebleed precipitants is also important in the overall management of people with HHT. Despite a battery of potential interventional, 
surgical, and medical approaches (4), HHT patients are commonly iron deficient and/or anaemic because replacing iron lost through recurrent haemorrhage demands very high iron intakes (3). In a recently surveyed international group, 273 of 1,288 (21.2\%) had received iron infusions and $396(30.8 \%)$ had received blood transfusions, $105(8.1 \%)$ on at least 10 occasions (5). Additionally, arterial rate bleeds can cause acute haemodynamic disturbances $(3,4,6,7)$; epistaxis severity is a major predictor of reduced quality of life (8-11); and epistaxis is the primary outcome measure in nine of ten clinical trials of new therapeutic agents recruiting in HHT (12).

Understanding what provokes HHT nosebleeds at particular times offers insights into haemorrhagic precipitants, and potential strategies to limit healthcare demands, improve quality of life, and optimise clinical trial design. HHT nosebleeds are highly variable, and generally difficult to predict. However, in recent studies by our group, HHT patients reported that nosebleeds could be precipitated by certain food groups $(13,14)$. Spontaneously volunteered precipitant foods included red wine, spices, chocolate, coffee, berries, oily fish, and other food items that contain high levels of naturally occurring salicylates and other anti-platelet agents (13). We hypothesised that for some people, suggesting dietary changes may also detrimentally impact on their quality of life, and therefore examined nosebleed relationships with dietary supplements that may be more readily modified.

\section{Materials and Methods}

\subsection{Ethics}

The study was given a favourable Ethics opinion by the London Wandsworth Research Ethics Committee (11/ $\mathrm{H} 0803 / 8)$, and all participants gave written informed consent.

\subsection{Survey methodology}

Based on previous experience from pilot study numbers, an unselected group of 50 patients with HHT attending our tertiary care clinic were recruited into a blood sample and questionnaire-based study during two study periods to coincide with recruiter availability, April-September 2011, and March-May 2013. Inclusion criteria were a definite diagnosis of HHT using the Curaçao Criteria (15) and not residing in the same household as another study participant. Within this period, $\sim 180$ eligible patients were approached by letter or in person. All recruited participants recorded nosebleed severity by the validated Epistaxis Severity Score (ESS) (16). This rates nosebleed severity on a scale of $0-10$, with the minimal important difference recently identified as 0.71 (17). Dietary supplement intake was assessed in an unbiased manner on the final page of the validated European Prospective Investigation into Cancer and Nutrition (EPIC) Food Frequency Questionnaire (18). Participants were asked "Have you taken any vitamins, mineral, fish oils, fibre or other food supplements during the past year?" and then asked to detail the brand, strength, amount and frequency ( 6 frequency options were provided).

\subsection{Haematological evaluations}

Blinded to epistaxis severity scores and supplement use, full blood count, prothrombin time (PT), activated partial thromboplastin time (APTT), fibrinogen concentrations, and biochemical analyses were measured as part of routine clinical care. Blood samples were obtained by a professional phlebotomist in the early to mid-afternoon, and centrifuged to obtain platelet-rich plasma (PRP) and platelet poor plasma (PPP). Blinded to supplement use and other blood results, PRP was used to determine platelet aggregation to freshly-prepared adenosine diphosphate (ADP), by a Helena Aggregometer.

\subsection{Statistical analysis}

Statistical analyses were performed using GraphPad Prism 6 (GraphPad Software, San Diego, CA). Participants were categorised by supplement use as described in the text. Where there were three categories (e.g. no supplement use, iron supplement, and non iron supplement use; or no supplement use, fish oil and non fish oil supplement use) $p$ values were calculated using Kruskal Wallis. Where there were two categories (e.g. supplements use, or no use of supplements), $p$ values were calculated using Mann Whitney.

\section{Results and Discussion}

The 50 study participants ranged in age from 18 to 72 (median 53) years with an even gender distribution. Haemoglobin ranged from 5.9 to 17.8 (median 14.1) g/ $\mathrm{dL}$, with higher values in participants with hypoxaemia (low blood oxygen levels) induced by pulmonary arteriovenous malformations (19) that affect at least $50 \%$ of people with HHT (20).

Of the 50 participants, 24 (48\%) had used dietary supplements in the previous year. Twelve of the HHTaffected study participants used iron supplements which would be fully appropriate given their high iron requirements, but 18/50 (36\%) also used between 1-3 non-iron containing supplements (Figure 1). There were no evident gender differences, but compared to the 26 study participants using no supplements (median age 53, IQR 40, 63 years), there were trends for iron supplement users to be younger (median age 47 , IQR $39.5,62.3$ years) and non-iron supplement users to be older (median age 58.5, IQR 49.5, 66 years). 


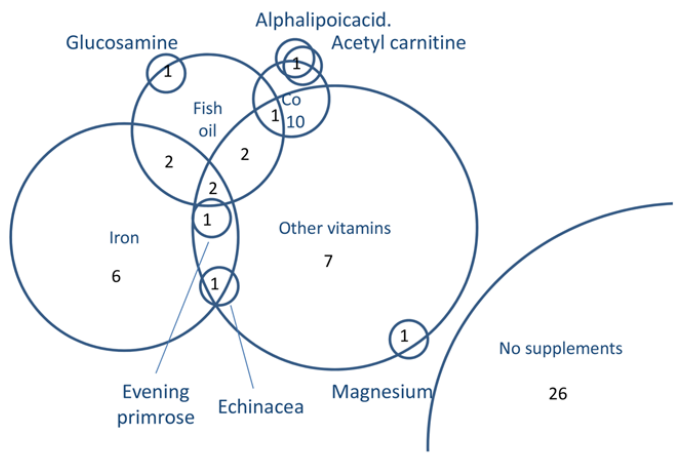

Figure 1. Dietary supplement use by 50 HHT patients. Venn diagram indicating overlapping use of dietary supplements, with circles to scale according to number of participants ingesting the item in the previous year.

The cohort demonstrated the full spectrum of nosebleed severity with epistaxis severity scores ranging from 0 (none) to 9.1 out of 10 (median 3.9; IQR 2.5, 5.0). The highest ESS of 9.1 was observed in two supplement users- one used fish oil and vitamins, and one used iron (Figure 2A). Overall, HHT patients with more nosebleeds tended to use iron supplements: the median ESS in the 38 non-iron users combined was 3.2 (IQR 1.8, 4.8) compared to 4.7 (IQR 4.0-5.2) in the 12 iron supplement users ( $p=0.012$ by Mann Whitney).

The difference in nosebleeds between the iron and non-iron using HHT cohorts substantially exceeded the minimal important difference for the epistaxis severity score (17), and corresponded to the difference between one nosebleed per week, typically lasting 1 to 5 minutes, and an average of one a day, typically lasting 6-15 minutes (16). For a male, the haemorrhageadjusted iron requirement (HAIR) (3) would increase from 9.1 to $35 \mathrm{mg} /$ day, compared to the UK recommended nutrient intake (RNI) of $8.7 \mathrm{mg}$ of iron per day (21).

However, the HHT patients using iron supplements had higher serum iron concentrations than the other study participants (Figure 2B), and despite their more frequent nosebleeds, were able to maintain their red blood cell indices (Figure 3). There was no discernable trend in mean corpuscular volume (MCV) or mean corpuscular haemoglobin $(\mathrm{MCH})$, indices that are generally unaffected by the secondary erythrocytosis observed in hypoxaemic patients with PAVMs (19). There were only possible trends towards lower haemoglobin and haematocrit values (Figure 3).

As noted in Figure 1, 18/50 (36\%) study participants used non-iron containing supplements. These were most commonly vitamins $(N=15)$ or fish oil $(N=8)$, and included glucosamine, coenzyme Q10, alpha lipoic acid, echinacea, magnesium, and evening primrose. The one individual using echinacea had stopped taking it, because it seemed to make their nosebleeds worse.

Fish oil supplements are known to have anti-platelet activity (22-25). In the cohort as a whole, epistaxis
A
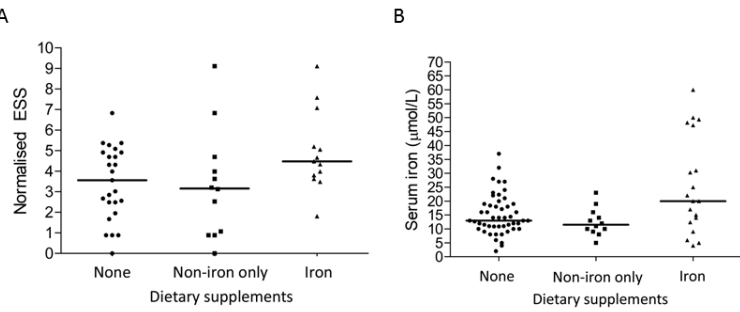

Figure 2. Nosebleeds and serum iron, categorised by dietary supplement use. (A) Epistaxis severity score (ESS): The ESS ranges from zero (no nosebleeds) to 10. As outlined in Figure 1, the non iron supplements comprised vitamins $(N=$ $15)$, fish oil $(N=8)$, glucosamine, coenzyme Q10, alpha lipoic acid, echinacea, magnesium, and evening primrose. Median values indicated. 3 group comparison, $p=0.075$ by Kruskal Wallis. (B) Serum iron in the 50 study participants. Note that the institutional normal range is $7-27 \mu \mathrm{mol} / \mathrm{L}$. Median values indicated. Overall Kruskal Wallis $p$-value $=0.033$. Data from all 50 study participants.
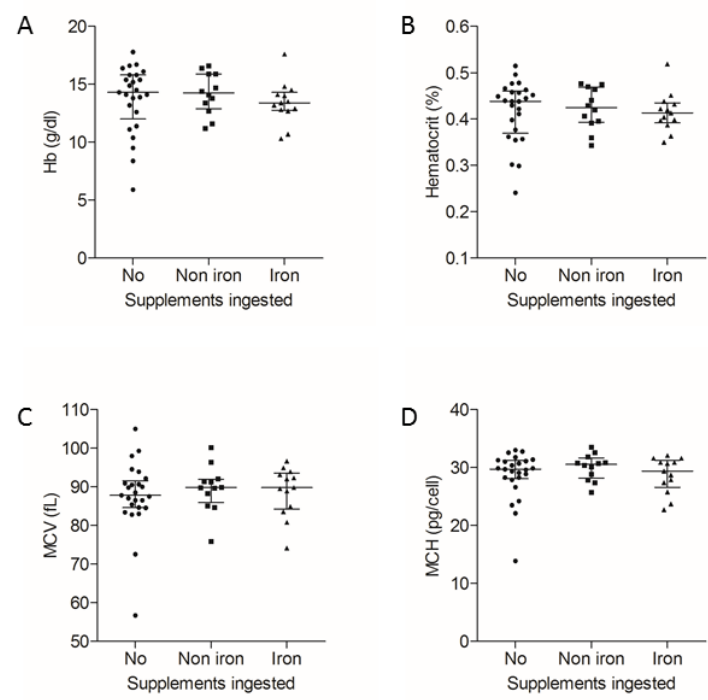

Figure 3. Red blood cell indices, categorised by dietary supplement use. (A) Haemoglobin ( $\mathrm{Hb}$, Kruskal Wallis $p=$ 0.41 ); (B) Haematocrit (Kruskal Wallis $p=0.62$ ); (C) Mean corpuscular volume (MCV, Kruskal Wallis $p=0.69$ ); (D) Mean corpuscular haemoglobin concentration $(\mathrm{MCH}$, Kruskal Wallis $p=0.66$ ). Median and interquartile range indicated. Data from all 50 study participants.

severity scores did not differ between users of fish oils and any other group (data not shown). However, subtle differences emerged examining indices related to platelets in blood from fish oil users compared to non users of fish oils. Platelet counts were lower in supplement users ( $p=0.037$, Figure 4A), while serum fibrinogen, which can act as a circulating protein for platelet aggregation (Figure 4B), also tended to be lower in fish oil supplement users $(p=0.07)$. For a subgroup of 11 patients, 'selected' based purely on dates of attendance at clinic when the study was in progress, platelet aggregation studies were undertaken and provided provocative, preliminary findings: Within this very small sample, although there was no apparent difference in platelet aggregation to ADP between iron/ 
A

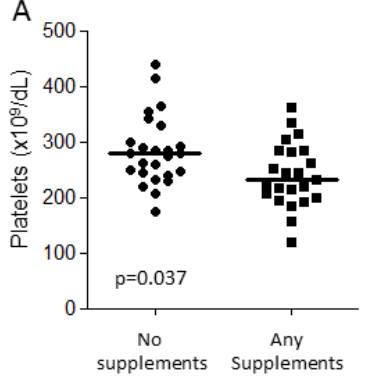

B

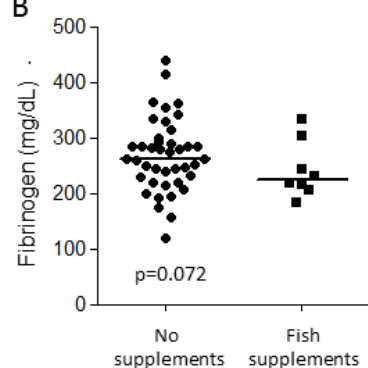

$\mathrm{C}$

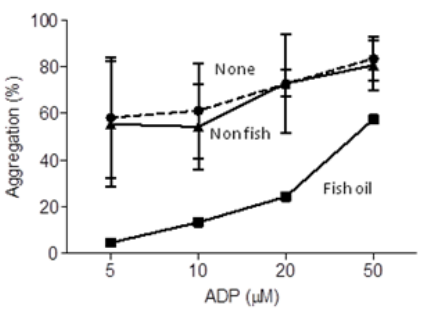

Figure 4. Coagulation and platelet aggregation. (A) Platelet counts and (B) Fibrinogen concentrations, in the 50 study participants, categorised by supplement use, median values indicated. There was no apparent difference in routine coagulation screens (prothrombin time, activated partial thromboplastin time, data not shown). (C) Platelet aggregation to stated concentrations of adenosine diphosphate (ADP) in the subgroup of 11 participants undergoing platelet aggregation studies, categorised by ingestion of no supplements ('None', $N=7)$, supplements other than fish oil ('non fish', $N=3$ ), and fish oil $(N=1)$. Means and standard deviations illustrated.

other vitamins, and non supplement users (Figure 4C), the one individual using fish oil demonstrated markedly reduced platelet aggregation to ADP (Figure 4C).

The strengths of the study were the use of validated questionnaires to capture epistaxis severity and dietary supplement intake, and the evaluation of a patient population able to recognise and report their haemorrhagic losses in a quantifiable manner. It is clearly a small study that should be repeated in larger HHT cohorts, and the observational nature means it is difficult to infer causality, but we believe it does allow helpful conclusions to be drawn.

First, it was surprisingly common for people with HHT to use dietary supplements. The non-iron supplements were generally instituted by the patients themselves. The supplements were not recorded on communications to us, and we suspect, were not recognised within standard clinical care pathways.

Second, the supplements which were self-prescribed included fish oils with recognised anti-platelet activity. The study participants appeared to demonstrate subtle differences in platelet activity, although differences in epistaxis scores did not emerge in the small cohorts. High proportions of HHT patients avoid therapeutic antiplatelet and anticoagulant agents, often on medical advice (26). It is not known whether any potential antiplatelet activity for example, from Omega-3 compounds (22-25), would be sufficient to exacerbate HHT nosebleeds in the subgroup of individuals who report that their HHT nosebleeds are exacerbated by aspirin and/or clopidogrel (26). We are however, unaware of any medical recommendation for their use in haemorrhagic disorders, and in the clinic, find patients to be surprised and concerned that these "healthy supplements" may act as natural blood thinners.

Third, within this small observational sample, despite having worse nose bleeds, iron supplement users had higher serum iron than non iron users, and were able to maintain their baseline haemoglobin and red cell indices. This would support data from other sources suggesting oral iron absorption is appropriate in people with HHT (i.e. enhanced in iron-deficiency (3)). It may be of concern however, that for five iron users, serum iron concentrations were at least twice the upper limit of normal (Figure 2B), in keeping with recent data from a healthy volunteers study (5). One in 20 HHT iron users report nosebleeds are worse after iron treatments $(5,27)$, and recent data suggest one plausible biological explanation through activation of endothelial DNA damage response pathways by $10 \mu \mathrm{M}$ iron (28), an order of magnitude lower than examined in recent iron toxicity studies $(29,30)$.

In conclusion, this study highlights how frequently people with HHT self-medicate with dietary supplements that may influence nosebleed (epistaxis) severity and platelet function. The scale of use, and potential of "natural health supplements" to exacerbate nosebleeds has not been appreciated previously in HHT. We suggest management of people with HHT and other haemorrhagic disorders should include a discussion of dietary supplements, and particularly the fish oil supplements that have recognised antiplatelet activity (22-25). For individuals with troublesome haemorrhage or iron deficiency, a trial of cessation of non-iron containing supplements may be indicated unless there has been clear benefit from their use.

\section{Acknowledgements}

This study received support from the National Heart and Lung Institute's BSc Project Funds (for B.C.), King's College London (for H.F.), and donations from families and friends of HHT patients. The authors also acknowledges support from the NIHR Biomedical Research Centre Funding Scheme (Imperial BRC). The funders played no role in the design or conduct of the study; collection, management, analysis, or interpretation of the data; preparation, review, or approval of the manuscript; or decision to submit the manuscript for publication. We thank Gillian Angus for advice on platelet aggregation measurements, and the patients for their willing participation in these studies. 


\section{References}

1. Shovlin CL. Hereditary haemorrhagic telangiectasia: Pathophysiology, diagnosis and treatment. Blood Rev. 2010; 24:203-219.

2. McDonald J, Wooderchak-Donahue W, VanSant Webb C, Whitehead K, Stevenson DA, Bayrak-Toydemir P. Hereditary hemorrhagic telangiectasia: Genetics and molecular diagnostics in a new era. Front Genet. 2015; 6:1.

3. Finnamore H, Le Couteur J, Hickson M, Busbridge M, Whelan K, Shovlin CL. Hemorrhage-adjusted iron requirements, hematinics and hepcidin define hereditary hemorrhagic telangiectasia as a model of hemorrhagic iron deficiency. PLoS One. 2013; 8:e76516.

4. Rimmer J, Lund VJ. Hereditary haemorrhagic telangiectasia.. Rhinology. 2015; 53:129-34.

5. Shovlin CL, Gilson C, Busbridge M, et al. Can iron treatments aggravate epistaxis in some patients with hereditary hemorrhagic telangiectasia? Laryngoscope. 2016; 10.1002/lary.25959

6. Hunter BN, Timmins BH, McDonald J, Whitehead KJ, Ward PD, Wilson KF. An evaluation of the severity and progression of epistaxis in hereditary hemorrhagic telangiectasia 1 versus hereditary hemorrhagic telangiectasia 2. Laryngoscope. 2016; 126:786-790.

7. Folz BJ, Tennie J, Lippert BM, Werner JA. Natural history and control of epistaxis in a group of German patients with Rendu-Osler-Weber disease. Rhinology. 2005; 43:4046.

8. Loaec M, Moriniere S, Hitier M, Ferrant O, Plauchu $\mathrm{H}$, Babin E. Psychosocial quality of life in hereditary haemorrhagic telangiectasia patients. Rhinology. 2011; 49:164-167.

9. Geirdal AØ, Dheyauldeen S, Bachmann-Harildstad G, Heimdal K. Quality of life in patients with hereditary hemorrhagic telangiectasia in Norway: A population based study. Am J Med Genet A. 2012; 158A:1269-1278.

10. Merlo CA, Yin LX, Hoag JB, Mitchell SE, Reh DD. The effects of epistaxis on health-related quality of life in patients with hereditary hemorrhagic telangiectasia. Int Forum Allergy Rhinol. 2014; 4:921-925.

11. Ingrand I, Ingrand P, Gilbert-Dussardier B, Defossez G, Jouhet V, Migeot V, Dufour X, Klossek JM. Altered quality of life in Rendu-Osler-Weber disease related to recurrent epistaxis. Rhinology. 2011; 49:155-162.

12. Clinical Trials.gov. www.clinicaltrials.gov (accessed February 4, 2016).

13. Silva BM, Hosman AE, Devlin HL, Shovlin CL. Lifestyle and dietary influences on nosebleed severity in hereditary hemorrhagic telangiectasia. Laryngoscope. 2013; 123:1092-1099.

14. Elphick A, Shovlin CL. Relationships between epistaxis, migraines, and triggers in hereditary hemorrhagic telangiectasia. Laryngoscope. 2014; 124:1521-1528.

15. Shovlin CL, Guttmacher AE, Buscarini E, Faughan M, Hyland R, Westermann CJJ, Plauchu H. Diagnostic criteria for hereditary hemorrhagic telangiectasia (Rendu-OslerWeber syndrome). Am J Med Genet. 2000; 91:66-67.

16. Hoag J, Terry P, Mitchell S, Reh D, Merlo C. An epistaxis severity score for hereditary hemorrhagic telangiectasia. Laryngoscope. 2010; 120:838-843.

17. Yin LX, Reh DD, Hoag JB, Mitchell SE, Mathai SC, Robinson GM, Merlo CA.The minimal important difference of the epistaxis severity score in hereditary hemorrhagic telangiectasia. Laryngoscope. 2015; doi 10.1002/lary.25669

18. International Agency for Research on Cancer and World Health Organisation. European Prospective Investigation into Cancer and Nutrition. 2010; British Medical Association; available at http://epic.iarc.fr/about.php (accessed February 4, 2016).

19. Santhirapala V, Williams LC, Tighe HC, Jackson JE, Shovlin CL. Arterial oxygen content is precisely maintained by graded erythrocytotic responses in settings of high/normal serum iron levels, and predicts exercise capacity: An observational study of hypoxaemic patients with pulmonary arteriovenous malformations. PLoS One. 2014; 9:e90777.

20. Shovlin CL. Pulmonary arteriovenous malformations. Am J Respir Crit Care Med. 2014; 190:1217-1228.

21. Dietary reference values for food energy and nutrients for the United Kingdom. Report of the Panel on Dietary Reference Values of the Committee on Medical Aspects of Food Policy. Rep Health Soc Subj (Lond). 1991; 41:1210.

22. Dyerberg J, Bang HO. Haemostatic function and platelet polyunsaturated fatty acids in Eskimos. Lancet. 1979; 2:433-435.

23. Moertl D, Berger R, Hammer A, Hutuleac R, Koppensteiner R, Kopp CW, Steiner S. Dose-dependent decrease of platelet activation and tissue factor by omega-3 polyunsaturated fatty acids in patients with advanced chronic heart failure. Thromb Haemost. 2011; 106:457-465.

24. Cohen MG, Rossi JS, Garbarino J, Bowling R, MotsingerReif AA, Schuler C, Dupont AG, Gabriel D. Insights into the inhibition of platelet activation by omega-3 polyunsaturated fatty acids: Beyond aspirin and clopidogrel. Thromb Res. 2011; 128:335-340.

25. Stanger MJ, Thompson LA, Young AJ, Lieberman HR. Anticoagulant activity of select dietary supplements. Nut Rev. 2012; 70:107-117.

26. Devlin HL, Hosman AE, Shovlin CL. Antiplatelets and anticoagulants in hereditary hemorrhagic telangiectasia. New Engl J Med. 2013; 368:876-878.

27. Shovlin CL, Patel T, Jackson JE. Pulmonary AVM embolisation reported to improve nosebleeds by a subgroup of patients with hereditary haemorrhagic telangiectasia. ERJ Open Res. 2016. doi 10.1183/23120541.00035- 2016

28. Mollet IG, Patel D, Govani FS, Giess A, Paschalaki K, Periyasamy M, Lidington EC, Mason JC, Jones MD, Game L, Ali S, Shovlin CL. Low dose iron treatments induce a DNA damage response in human endothelial cells within minutes. PLoS One. 2016;11:e0147990.

29. Chan S, Chen MP, Cao JM, Chan GC, Cheung YF. Carvedilol Protects against Iron-Induced Microparticle Generation and Apoptosis of Endothelial Cells. Acta Haematol. 2014; 132:200-210.

30. Kartikasari AE, Georgiou NA, Visseren FL, van KatsRenaud H, van Asbeck BS, Marx JJ. Endothelial activation and induction of monocyte adhesion by non transferrin-bound iron present in human sera. FASEB J. 2006; 20:353-355.

(Received March 29, 2016; Revised April 5, 2016 ; Accepted April 7, 2016) 